\title{
Berichtigung \\ Die Röntgenkleinwinkelstreuung von dichtgepackten kolloiden Systemen
}

\author{
I. Teil
}

Von G. Porod, Graz

Kolloid-Z. 124, 83 (1951)

Seite 95 links 7. Zeile im 3. Absatz - lies: Kleinstwinkelstreuung statt „Kleinwinkelstreuung“ Seite 95 rechts 1. Zeile im 3. Absatz - lies: des ersten Faktors statt ,zweiten“

$$
\text { Seite } 96 \text { Formel }[1] \text { - lies: }\left(\frac{\sin s d / 2}{s d / 2}\right)^{2} \text { statt }\left(\frac{\sin s d / 2}{s d / s}\right)^{2}
$$

Seite 101 links 3. Zeile nach Formel [18]; rechts 5. Zeile - lies: „, $l^{\prime \prime}$ statt ,I “ Seite 106 rechts 6. Zeile nach Formel [30]: wio oben

$$
\begin{aligned}
& \text { II. Teil } \\
& \text { Kolloid-Z. 125, } 51 \text { (1952) } \\
& \text { Seite } 113 \text { links Formel [18] lies: }\left(\frac{d H}{d x}\right)_{0} \text { statt }\left(\frac{d H}{d x}\right)_{c} \\
& \text { Seite } 11 \text { כ links Formel [24] lies: .... }=\frac{8 \pi w w}{R \lambda} \frac{\int_{0}^{\infty} \hat{\imath} z d z}{\int_{0}^{\infty} \hat{\imath} d z} \text { statt } \frac{8 \pi}{R \lambda} \frac{\int_{0}^{\infty} \hat{\imath} z d z}{\int_{0}^{\infty} \hat{\imath} d z}
\end{aligned}
$$

Herrn Prof. Dr. O. Kratky bin ich für Korrekturhinweise zu bestem Dank verpfliohtet.

\section{Bücherbesprechungen}

Industrielle Elektronik. Von R. Kretzmann - Hamburg. 232 Seiten mit 234 Abb. (Berlin -Borsigwalde, 1952, Verlag für Radio-Foto-Kinotechnik GmbH.), Preis: DM. 12,50.

Insbesondere in USA ist nicht nur die Entwicklung und Anwendung der Elektronenröhren-Technik durch die hente schon wesentlich größere Verbreitung des Fernsehens stärker Allgemeingut, es hat sich dort auch die Elektronik als Hilfe bei Rationalisierung, Steuerung und Überwaohung technischer Fortschritte schon weitgehend eingebürgert. Für zahlreiche Probleme, die bei uns mit optischen oder sogar noch rein mechanischen Mitteln gelöst werden, wurden die Vorteile der Verwendung der Elektronik erkannt und ausgenutzt. Ziel des vorliegenden Buches ist es, diesen Zweig derTechnik und die Erfahrungen hierbei auch dem deutschen Leser aus Industrie und Labor an Hand von Beispielen vorzuführen und insbesondere die zum Teil noch herrschenden Vorurteile beseitigen zu helfen.

Diese Aufgabenstellung ist übersichtlich gelöst, indem in einem 1. Teil die speziell zu diesen Zwecken entwickelten Elektronenröhren einzeln ihrem Aufbau und ihrer Wirkungsweise nach behandelt werden, während der 2. Teil die Anwendungsmöglichkeiten bringt. Da die Anforderungen hierbei anders als in der Nachrichtentechnik sind, wurden Spezialröhren verschiedenster Art, insbesondere für Schaltzwecke auch größerer Leistung, entwickelt, die allgemein noch weniger bekannt sind. Verfasser ist infolge seiner eigenen Mitarbeit besonders prädestiniert, gerade dieses Gebiot dem Leser darzulegen. Bei der Anwendung ist der Hauptwert auf Relaiszwecke, Regeltechnik und Steuerungsvorgänge gelegt. Vielleioht wäre es wünschenswert, wenn hier in einer späteren Ergänzung die Darstellung der ebenfalls in derUSA schon wesentlich verbreiteteren Ausnutzung der Elektronik für Meß- und Registrierzwecke angeschnitten werden könnte. Ref. denkt hierbei an registrierende
Spektrographen, elektrische Waagen und Kraftmeßsysteme.

Das vorliegende Buch stellt ein wertvolles Hilfsmittel für den Techniker und Ingenieur dar, zumal durch detaillierte Behandlung von Schaltungen, in einem Anhang Röhrendaten, sowie im Text Kennlinien und Nomogramme gebracht werden, so daB die Grundlagen für Anwendung auf neue Probleme gegeben sind. Der Verlag sorgte für vorzügliche Ausstattung.

F. H. Müller (Marburg/L.)

Kalorisch-chemische Rechenaufgaben. Von M. v. Stackelberg-Bonn. 71 Seiten und 2 Abb., (BerlinGöttingen-Heidelberg 1952, Springer-Verlag). Preis: geb. DM. 5.80 .

Die Aufgabensammlung enthält 31 elementare, aber sehr instruktive Aufgaben aus der ohemischen Thermodynamik mit ausführlichen Lösungen und Diskussionen. Das Zahlenmaterial ist ausschließlich dem ,Taschenbuch für Chemiker und Physiker" von J. D'Ans und E. Lax entnommen. Dadureh wird dem Studierenden die vielseitige Verwendbarkeit der betreffenden Tabellen gezeigt und dem Irrtum vorgebengt, daB zu physikalisch-chemischen Rechnungen unbedingt große Tabellenwerke erforderlich seien. Durch die Beschränkung der Fragestellung anf die engere chemische Thermodynamik werden nur wenige Gleichungen gebraucht. Die Zahl der Formeln ließe sich allerdings noch weiter einschränken, wenn auf die überflüssige und den Anfänger nur verwirrende doppelte Symbolik ( $W$ und $\Delta H, N$ und $\Delta G$ ) verzichtet wïrde. Im übrigen aber hält der Ref. das Büchlein für hervorragend geeignet, dem jungen Chemiker die Scheu vor der angeblich so schwerverständlichen und unanschaulichen Thermodynamik überwinden zu helfen.

K. Neumann (Gießen) 